\title{
INTERNAL ELECTROSTATIC TRANSDUCTION FOR BULK-MODE MEMS RESONATORS
}

\author{
Sunil A. Bhave and Roger T. Howe \\ Berkeley Sensor \& Actuator Center, 497 Cory Hall, University of California, Berkeley, CA 94720
}

\begin{abstract}
This paper demonstrates a new approach to electrostatic drive and detection of bulk acoustic resonators in which the electrode-gaps are filled with a high dielectric constant material. Internal electrostatic transduction has much higher efficiency than air-gap electrostatic transduction for bulk-mode resonators, which results in improved electrical performance. As a proof-of-concept, we demonstrate this phenomenon by electrostatic actuation of a $1.9 \mathrm{GHz}$ AlN $(\kappa \sim 9)$ film bulk acoustic resonator (FBAR).

\section{INTRODUCTION}

Surface micromachining technology supports fabrication of multifrequency, electrostatically transduced lateral bulk resonators. A single mask can include multi-frequency filters, oscillators and mixers. However, lateral bulk acoustic resonators have very large motional resistance due to reduced transducer area [1] and inefficient air-gap electrostatic transduction (compared to piezoelectric transduction [2]). Creative approaches to increasing transducer area include forming a coupled array of resonators [3] and large diameter bulk annular ring resonators [4]. However, to reach motional resistances on the order of $50 \Omega$, we would need a coupled array of 100 resonators or a $400 \mu \mathrm{m}$ diameter ring resonator. The signal routing challenges for these structures will be daunting at $\mathrm{GHz}$ frequencies and the chip area occupied by these resonator designs will be larger than an FBAR (which has motional resistance of $2 \Omega$ ).
\end{abstract}

The electrostatic force and motional current for a parallel-plate electrostatic transducer are:

$$
f=V_{D C} \cdot \frac{\varepsilon_{0} \cdot A}{g^{2}} \cdot v_{i n} \quad ; \quad i=V_{D C} \cdot \frac{\varepsilon_{0} \cdot A}{g^{2}} \cdot \omega \cdot x
$$

Both terms are proportional to the permittivity of capacitor dielectric ( $\varepsilon_{0}$ for air or vacuum). We propose to fill the electrode gaps of the bulk acoustic resonators with a dielectric material

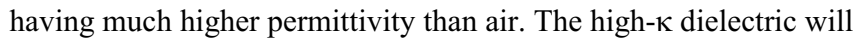
enhance both the force density of the electrostatic actuator as well as the sense capacitance, thereby reducing the motional resistance of these resonators by $\kappa^{2}$.

Bouwstra et al demonstrated that audio-frequency cantilever beams can be driven and sensed using silicon nitride dielectric capacitors embedded in a silicon resonator [5]. The resonator made use of Poisson's ratio to convert applied strain perpendicular to the beam's thickness into strain along the beam, which coupled into the fundamental bending mode. The approach was deemed inefficient because air-gap capacitive transduction provided larger displacement, the preferred performance metric at that time.

\section{BULK-MODE INTERNAL ELECTROSTATIC TRANSDUCTION}

Bulk-mode resonators have significantly different design requirements compared to flexural resonators. These resonators typically have displacements on the order of a few nanometers. In

Travel support has been generously provided by the Transducers Research Foundation and by the DARPA MEMS and DARPA BioFlips programs. principle, we can enhance the transduction efficiency of bulk resonators by filling the air-gaps with a low Young's modulus, high- $\kappa$ dielectric material. A more practical approach would be to find a dielectric with similar acoustic velocity as the resonator material and 'build-in' an internal electrostatic transducer at the maximum strain 'anti-nodes' rather than the maximum displacement nodes. This approach would minimize bulk energy losses due to acoustic velocity mismatch and optimize transduction efficiency of the resonator. $\mathrm{TiO}_{2}$ with relative permittivity $\kappa \sim 80$ and bulk acoustic velocity $7900 \mathrm{~m} / \mathrm{s}$ is an attractive material for this purpose.

In order to benchmark the performance of the internal electrostatic transducer, we evaluate its performance in a $3^{\text {rd }}$ overtone lateral bulk acoustic resonator. This class of resonators has been demonstrated with air-gap electrostatic $[\mathbf{1 , 4}]$ and piezoelectric transduction [2]. The $3^{\text {rd }}$ overtone can be excited and detected by introducing layers of $\mathrm{TiO}_{2}$ at the two anti-nodal planes, as shown in Figure 1.

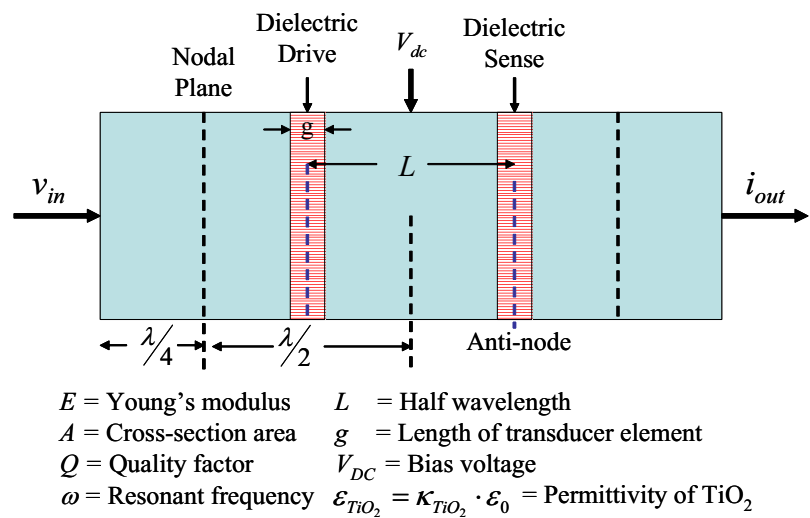

Figure 1. Schematic of electrostatically transduced $3^{\text {rd }}$ overtone bulk acoustic resonator

The motional resistance of this resonator is

$$
\frac{1}{R_{\text {electrostatic }}} \approx \frac{V_{D C}^{2} \cdot \kappa_{T_{i} O_{2}}^{2} \cdot \varepsilon_{0}^{2} \cdot A^{2}}{g^{4}} \cdot \frac{\omega \cdot Q}{E \cdot A / L}
$$

By replacing the electrode-gap with $\mathrm{TiO}_{2}$ at the antinodes, we can reduce the motional resistance by $\kappa^{2}=6,400$.

A $14 \mathrm{MHz}$ bulk acoustic resonator with $1 \mu \mathrm{m}$ air-gap electrostatic transducers has a motional resistance of $590 \mathrm{k} \Omega[1]$. The $3^{\text {rd }}$ harmonic of an identical resonator with $\mathrm{TiO}_{2}$ dielectric transduction would have a motional resistance of $275 \Omega$. Similarly, the motional resistance of the $1.2 \mathrm{GHz} 3^{\text {rd }}$ harmonic ring resonator [4] would scale down from $282 \mathrm{k} \Omega$ to $44 \Omega$.

\section{ELECTROSTATIC EXCITATION OF AN FBAR}

We used Agilent Technologies' AIN FBAR [6] to demonstrate internal electrostatic transduction. AIN has a relative permittivity of $\kappa \sim 9$ and the resonator has a mechanical quality factor $Q \sim 1350$ 
at a resonant frequency of $f_{0}=1.92 \mathrm{GHz}$. Electrostatic force is quadratic; therefore we can actuate the FBAR with an input signal at half the resonant frequency (Figure 2). This ensures that there is no piezoelectric actuation of the resonator. A low-pass-filter was added to prevent any harmonics from the RF synthesizer from reaching the input electrode.

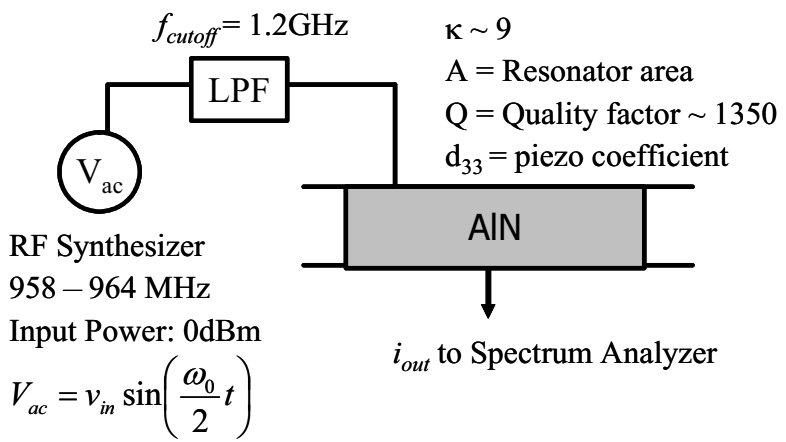

Figure 2. Test equipment setup for half-frequency measurement. The Spectrum Analyzer is set to MAX_HOLD as the synthesizer frequency is swept near half-resonance frequency.

Electrostatic actuation will generate stress in the resonator at the resonant frequency:

$$
T_{\text {electrostatic }}\left(f_{0}\right)=\frac{1}{4} \cdot \kappa_{A l N} \cdot \varepsilon_{0} \cdot \frac{v_{i n}^{2}}{t^{2}}
$$

This electrostatic stress generates dielectric displacement and results in piezoelectric displacement current:

$$
i_{\text {out }, \text { piezo }}\left(f_{0}\right)=\omega_{0} \cdot A \cdot Q \cdot d_{33} \cdot \frac{1}{4} \cdot \kappa_{A l N} \cdot \varepsilon_{0} \cdot \frac{v_{\text {in }}^{2}}{t^{2}}
$$

The output current also has an electrostatic component due to the quadratic electrostatic force. However, this component is extremely small compared to the piezo component due to the relatively large resonator thickness.

By sweeping the RF synthesizer frequency from $958 \mathrm{MHz}$ to $964 \mathrm{MHz}$ and using the MAX_HOLD function [7] on the 8562EC Spectrum Analyzer, we were able to construct the mechanical transfer function and extract $Q$ of the FBAR (Figure 3).

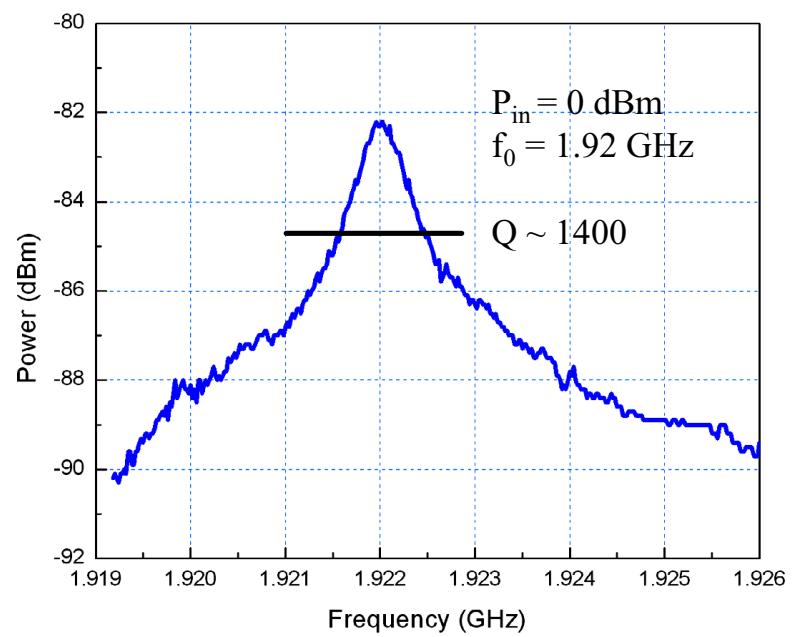

Figure 3. FBAR transmission spectrum obtained using halfresonance electrostatic actuation. $Q \sim 1400$ was extracted from the shape of the transfer function.

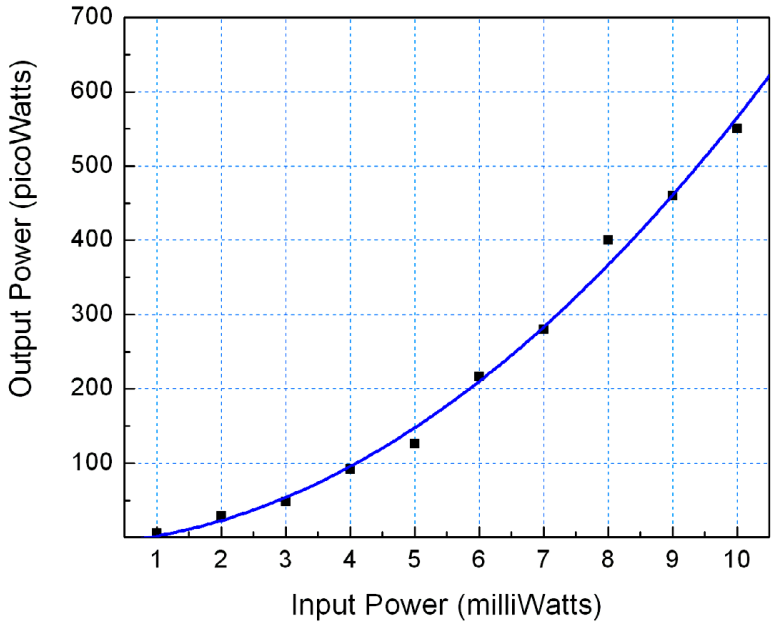

Figure 4. Output power is proportional to the square of the input power, verifying internal electrostatic actuation of the FBAR.

While the output current is due to piezoelectric effect, the mechanical motion of the FBAR is due to electrostatic stress. Hence, both the mechanical motion and output power are proportional to square of the input power (Figure 4).

The FBAR is a one-port device and hence is not suitable for electrostatic transduction. However, these two measurements provide preliminary experimental verification of internal electrostatic drive for bulk-mode resonators.

\section{CONCLUSION}

Internal electrostatic transducers using high- $\kappa$ dielectrics can achieve $\kappa^{2}$ higher efficiency than conventional air-gap transducers. This new approach will enable us to fabricate arrays of small footprint lateral bulk acoustic resonators with motional resistances $<1 \mathrm{k} \Omega$. It will also open up the opportunity to design microwave frequency resonators with reasonable motional resistances. As a proof-of-concept, we excited an FBAR at $1.92 \mathrm{GHz}$ with internal electrostatic actuation.

\section{ACKNOWLEDGMENT}

The authors wish to thank Dr. Dan Radack and the DARPA NMASP program, whose generous grant (\#N66001-00-1-8955) has made this research possible.

\section{REFERENCES}

1. T. Mattila, et al, "Micromechanical Bulk Acoustic Wave Resonator," 2002 Ultrasonics Symposium, pp. 945-948.

2. S. Humad, et al, "High Frequency Micromechanical Piezo-onSilicon Block Resonators," IEDM 2003, pp. 957-960.

3. M. Demirci, et al, "Mechanically Corner-Coupled Square Microresonator Array for Reduced Series Motional Resistance," Transducers 2003, pp. 955-958.

4. S.-S. Li, et al, "Micromechanical "Hollow-Disk" Ring Resonators," MEMS 2004, pp. 821-824.

5. S. Bouwstra, et al, "Excitation and Detection of Vibrations of Micromechanical Structures using a Dielectric Thin Film," Sensors and Actuators, 17 (1989), pp. 219-223.

6. R. Ruby, et al, "Ultra-Miniature High-Q Filters and Duplexers using FBAR Technology," ISSCC 2001, pp. 120-121.

7. J. Wang, et al, "1.14-GHz Self-Aligned Vibrating Micromechanical Disk Resonator," RFIC 2003, pp. 335-338. 